\title{
Complex System Theory and College English Teaching Developments
}

\author{
Liang Aimin ${ }^{1, *}$, Cong Rizen ${ }^{2}$ \\ ${ }^{1}$ School of Foreign Languages, University of Jinan, Jinan City, China \\ ${ }^{2}$ Room 704, No. 8 Building, No. 155-2, Yingxuongshan Road, Jinan City, Shandong Province, China \\ *Corresponding author: sfl_liangam@ujn.edu.cn
}

Received September 30, 2014; Revised October 13, 2014; Accepted October 20, 2014

\begin{abstract}
Complex systems are composed of elements or agents that are of many different types and that interact in different ways. Complex System Theory, originating in the fields of physics and mathematics, and also popularly known as Chaos Theory or Dynamic System Theory, attempts to describe the interactions of different elements and agents with the features of heterogeneity, dynamics, openness, adaptation, non-linearity, and sensitive dependence on initial conditions etc and supplies new perspectives to the researches of Applied Linguistics. This article tries to reveal the prospect of the application of Complex System Theory in College English Teaching of China with the purpose to explore effective approaches to its developments of the following elements: such as, needs analysis, teaching objectives, teaching materials, testing, teaching models and evaluations.
\end{abstract}

Keywords: complex system theory, college English, teaching developments

Cite This Article: Liang Aimin, and Cong Rizen, "Complex System Theory and College English Teaching Developments." American Journal of Educational Research, vol. 2, no. 10 (2014): 925-931. doi: 10.12691/education-2-10-12.

\section{Introduction}

In China, College English (CE) refers to the English course learned by all non-English major students, which is regarded as an integral part of higher education and a required course for most undergraduate students. It is not only a language course that provides basic knowledge about English, but also a capacity enhancement course that helps Chinese students to broaden their horizons and learn about different cultures in the world. Therefore, College English Teaching (CET) has become a research focus in China. Since the 1980s, CET has experienced several great reforms. Scientists and teachers did a lot of work to apply various modern teaching theories into CET and devoted a lot to the study of such specific elements as teachers (their roles and teaching styles), students (their roles, motivations and personal differences), teaching situations and teaching effects etc.. Up till now, Great achievements have been achieved on them. However, all the former researches only focused on the study of CET individual elements and they had no awareness that CET is an integrated complex dynamic system in which all the elements interact with each other, change all the time and adapt to the different needs of students and society.

Complex System Theory, which originated in the fields of physics and mathematics and later applied to biology, economics, engineering and computer science, has been regarded as one of the most advanced theories of 21st Century. Larsen Freeman, the famous USA's linguist and educator, first took the initiative to introduce the Complex
System Theory into Applied Linguistics and published lots of pioneering works on it, such as: Chaos/Complexity Science and Second Language Acquisition [8]; Language Acquisition and Language Use from a Chaos/Complexity Theory Perspective [9]; On the Complementarity of Chaos/ Complexity Theory and Dynamic Systems Theory in Understanding the second language Acquisition Process [11]; Complex System and Applied Linguistics [12] etc.. In her works, Larsen Freeman explains the key constructs: chaos, complexity, dynamic system, etc., demonstrates applications to areas of first and second language development, language teaching and discourse analysis etc., illustrates how complex system thinking can challenge established ideas. Since then, many researchers in western countries have rethought about some elements in language development, language acquisition, and language use which are seen as complex, non-linear, dynamic processes. However, in China, this new perspective has not aroused enough attention, and the Complex System Theory remains neglected, especially, in CET field. Therefore, this paper aims to reveal the prospect of the application of the Complex System Theory in CET by combing through its connotation and analyzing its features and try to explore the effective approaches to CET developments which mainly concern with the following elements: such as, needs analysis, teaching objectives, teaching materials, testing, teaching models and evaluations.

\section{Introduction of Complex System Theory}


Complex Systems Theory, also Chaos Theory or Dynamic System Theory, offered a compelling approach to describing and explaining some real-world phenomena in natural and social sciences for it has profound connotations.

\subsection{Connotations of Complex System Theory}

In broad sense, a system is produced by a set of components that interact in particular ways to produce some overall state or form at a particular point in time. Simply speaking, a system has elements that interact to form a connected whole. For example, all the followings are systems, some simple, and some complex: the time system, a traffic light system, a transport system, the solar system, a living cell, an ecological system, a grammatical system, a speech community and a city. Not all the exemplar systems above are "complex". Such as, the time and traffic light system are not complex but simple ones, since they have just a small number of elements of a single type, together with predictable patterns of behavior. In the time system, the component elements are seconds, minutes, hours and days etc. that follow each other in a strict order. Such a time sequence can not be influenced by other things in the real world so that it can be predicted. In the system of the traffic lights, there are usually three lights that appear in a fixed sequence: red; yellow; green. The three different colored lights are the elements of the system. Their appearance produces three permutations that are made use of in stopping and starting traffic. The traffic light system, largely unaffected by actions of drivers or other aspects of the environment, moves through the three stages, repeatedly and predictably. In the simple system, if we know the "rules" that elements follow in their interactions the future state of the system can be predicted, that is, in the time system, if we have passed Tuesday, we are sure to meet Wednesday next day. In the system of the traffic lights, if we see a red and yellow light, we can confidently predict that green light will follow.

However, complex systems, differing from simple systems, refer to systems with many different types of agents and elements which interact in different ways over time. Such systems are dynamic, open, non-linear, and adaptive. They change over time, sometimes steadily, while at other times, they change suddenly and dramatically. Through the sudden change, the system selforganizes, generating new, emergent, modes of behavior. They are generally unpredicted. Among the former examples, except the time system and traffic light system, all the rest systems belong to complex systems because similar features, such as, heterogeneity, dynamics, openness, adaptation, non-linearity, and sensitive dependence on initial conditions can be seen in each of these very different systems.

\subsection{Features of Complex System Theory}

Simply speaking, complex system is a heterogeneous, dynamic, open, non-linear and adaptive system [12]. So the features of complex system are the followings:

1) Heterogeneity: Complex systems have many different types of component or agents and elements and all of them are "heterogeneous". For example, in the forest ecosystem, there are many different kinds of component agents: animals, birds, insects, and people, while component elements would include trees, winds, rainfall, sunshine, air quality, soil, rivers. All these agents and elements, with different natures and types, interact with each and form interconnected processes: processes of growth and decay, of feeding and digestion, of mating and breeding etc.

2) Dynamics: In complex systems, everything is dynamic: not only do the component elements and agents change with time, giving rise to changing states of the system, but the ways in which components interact with each other also change with time. The changes are continuous and go on at each level. For example, any plant in nature grows continuously. It may slow down if the weather is cold, but growth continues. It may go through cycles of flowering and dying back, but growth is some sort still continues; a system of human activity or development is also dynamic at each level of social or human organization, from the socio-cultural, through the individual and down to the neural and cellular.

3) Openness: Complex systems are open systems, in that energy and matter can come into the system from outside to maintain non-equilibrium, an ordered state, whereas a closed system will reduce to a stable state or equilibrium. For example, in the transform system, when there is not too much traffic in a road system, a state of order will be maintained where the traffic flows freely from stating point to destination. If the system is open to more and more traffic, it will eventually reach equilibrium in the form of gridlock. If it is also "open" in the sense that more roads are constructed to provide for the increasing traffic, free-flowing order may be maintained. We must know clearly that a complex system, that is, an open, non-equilibrium and ordered system is in the 'stability in motion' or a 'dynamic stability ', not a stability that is static or fixed. A further example of this kind of dynamic stability might be the financial stability which an individual maintains over a period of months as money flows in and out of bank accounts.

4) Adaptation: The process in which a system adjusts itself in response to changes in its environment is adaptation, and systems that work in this way are called “complex adaptive system", an important class of complex systems. Take a swimmer floating in the sea or swimming pool as an example [12], the water is not by itself able to support a floating human body, but if the swimmer makes small movements of hands or feet, he or she can stay floating on the surface of the water indefinitely. In this example, the movements of the swimmer are adaptations made in response to the environment---to the need to prevent sinking.

5) Non-linearity: Non-linearity is a mathematical term, referring to change that is not proportional to input. A complex system is a nonlinear system in which the effect is disproportionate to the cause and the elements or agents are not independent, and relations or interactions between elements are not fixed but may change by themselves. Conversely, in a linear system, a cause of a particular strength results in an effect of equal strength, the relations among the elements and agents remain fixed, and change in the system is constant and proportionate. Imagine a linear transport system that contains roads, cars, buses, and the people who use them, so if the people use more cares the roads will be proportionately busier; if more people take buses, there will be proportionately fewer cars 
on the road. If more roads are built for the same number of cars, the roads will be proportionately less busy. The future of this linear system is completely predictable. Of course, the real life is not so linear! The new roads don't just spread out the same number of cars, but actually encourages more people to use it because of its convenience. It is likely that the new roads will be soon blocked with unexpected traffic jams. So we can say city transform system is a complex system because there are many non-linear changes in it.

6) Sensitive Dependence on Initial Conditions: A major reason for the unpredictable behavior of complex systems is their sensitive dependence on initial conditions or starting states. A slight change in initial conditions can have vast implications for future behavior. Indeed, the behavior of systems with different initial conditions, no matter how similar, diverges exponentially as time passes. A phenomenon known popularly as "the butterfly effect" exemplifies this feature and underscores the interdependence of all the components in the system. The butterfly effect is the notion that a tiny motion such as the flap of a butterfly's wings is sufficient to generate changes that amplify to produce a tornado on the other side of the world. That is, tiny differences in input could quickly become overwhelming differences in output.

In order to prove sensitive dependence on initial conditions, Larson-Freeman offered the following example in her book Complex Systems and Applied Linguistics. According to English grammar, if we begin our utterance with an article, it is likely that a noun will follow soon after. However, the fact show that such an inference is not always right. An example is the utterance that 'The old man the boat'. From the form itself, let alone its meaning, we are led to expect a noun to follow the article 'the' and the adjective 'old'. Thus, our initial reading of 'man' is as a noun. It is only when we reach the noun phrase 'the boat' that we realize that we have been wrong. At this point, we have to reanalyze 'the old' as a collective noun deriving from an adjective, which serves as the subject and 'man' here as the verb. Thus initial conditions can affect the life of the language, the prior discourse of language use, and even the interpretation a single utterance [12].

\subsection{Complex System Theory and CET}

In China, College English is a second language or a foreign language. Therefore, CET is closely connected with Second Language Acquisition (SLA) which has been considered to be a complex system by many scholars, such as: Diane Larsen-Freeman [8-14]; Paul Van Geert [16,17]; De Bot, Lowie and Verspoor [4,5,6]. Such an opinion encourages our College English teachers must study CET in complex system thinking and challenge some of its established ideas. Many proofs have shown that CET has much in common with a complex system.

Firstly, CET has the characteristic of heterogeneity. In CET, there are many different types of heterogeneous elements or agents including teachers, students, courses, and teaching situations etc.. All of them have their own features. They are interdependent, interacting with each other, and changing in different ways over time. For example, students can be regarded as a complex system in which they interact with teachers, courses, and learning situations. In the individual student system, there are also cognitive, psychological, emotional, and ecological subsystems which are developing and changing under their own influences which result in the students' individual differences.

Secondly, CET system is a dynamic complex system in which all the elements or agents are in continuous motion and the interactions among the elements are also changing over time. For example, the teaching objective of CET is always changing with social and economic development. The contents of College English course are dynamic in order to meet students' learning needs and the social needs. The CET materials are developing with the globalization and development of information technology. The teaching methods and evaluations are often reformed and improved so as to increase the teaching efficiency and catch up with the times' developments. Meanwhile, in the process of English learning, students' cognitive situation and the socio-cultural situations are combined together and continuously interacting to promote students' language developments and result in great changes. As for the individual College English learners, their ages, gender's roles, psychological, emotional, and ecological elements are all dynamic over time.

Thirdly, CET system is a non-linear system. That is to say, in CET, teaching does not cause learning and learning does not surely cause acquisition. That is the reason why teachers' great devotion to English teaching and students' hard work at English learning can not always result in the expected results. As we all know, language learning and acquisition is not a linear system because many other unpredicted factors affect them greatly. For instance, according to Meara's research in [15], measures of vocabulary learning in a second language show that learning begins quite slowly. Once a certain number of words are mastered, learning increases in rate until vocabulary size reaches some level that seems to serve the student well enough, and then the rate of learning slow down. That is to say, vocabulary size does not increase linearly over time. Another example is about the grammar mistakes that students make. They are also non-linear. Because having been corrected, the mistakes will not surely disappear forever and students will make the same mistakes again and again.

Fourthly, College English is an open, adaptive system because English is open to all sorts of influences, and is continually developing. In CE, teachers, students, curriculum, and learning environment needs to continually adapt to the changes in the learners, and the developments in society. The outside situation of CET is also an open and adaptive system which is influenced by the highest developing world. The teaching materials and methods should be open to the outside world and to absorb what students are most interested in. That is the reason why Chinese Education Department requires that every university and college should adapt their teaching objectives, teaching materials and testing to the students' learning and social needs and to explore the most effective teaching methods and evaluation systems to adapt to the students' practical English level.

Fifthly, CE also has one of the most essential characteristics of dynamical systems is what has been called the sensitive dependence on initial conditions, or the Butterfly Effect. In College English, there are many 
kinds of initial conditions to determine whether students can be successful or not in English learning. The initial conditions include students' birthplaces, initial interests, learning methods and learning contexts etc.. For example, in China, the problems in pronunciation can cause great alternatives in students' English level and proficiency. Such a phenomenon happened to both Southern students and Northern students because their local accents and Chinese phonetic alphabet affect their pronunciation so greatly that they cannot pronounce some of the English consonants correctly, such as: /r l/, / s s/, /s z/,/ $/ \theta$ ð/ /W v /, etc.. As a result, they fail in English listening and speaking and can not communicate with others effectively. Many facts also certificates that students' initial interests, their first English teachers and their early learning contexts can lead to quite different and even opposite outcomes in their English learning.

\section{Pedagogical Implication for CET}

A complex system perspective on CET highlights the interactions across interconnected elements, components in it. According to Brown's Systematic Curriculum Development Model in [[2], p. 29], there are several components in language curriculum development, that is, "needs analysis", “teaching objective”, "language testing”, "material development”, “language teaching”, and "program evaluation". Therefore, CET should be developed in the following five aspects.

\subsection{Heterogeneous CE Needs Analysis}

Needs Analysis (NA), also called Needs Assessment, refers to "the activities involved in gathering information that serves as the basis for developing a curriculum that meets the learning needs of a particular group of students" [[2], p. 35]. In needs analysis, there are many heterogeneous agents and elements to be concerned with, Such as: different categories of needs, people, questions, and instruments etc.. These heterogeneous elements interact with each other, change over time, and supply the believable data.

Firstly, before making needs analysis, the researchers should investigate different categories of needs. That is, heterogeneous. The most complete need analysis should contain the following main categories of needs: "objective needs", “subjective needs”, “target needs”, "learning needs" etc.. Objective needs refer to all factual information about the learner, that is, the "biographical data" .Subjective needs refer to the cognitive and affective needs of the learners in language learning. Target needs focus on the situations in which the target language is used, which is concerned with what the learner needs to do in the target situation and also named "social needs". Learning needs refer to what the learner needs to do in order to learn what they should learn in some curriculums.

Secondly, before any needs analysis can take place, the researchers must make it certain who will be involved in the needs analysis, that is, who will be the agents. Generally speaking, four different categories of people may become involved in a needs analysis: the target group, the audience, the needs analysts themselves, and the resource group. The target group is made up of those people about whom information will ultimately be gathered. In CET, The usual target groups are students, teachers and administrators. The audience for a needs analysis should encompass all people who will eventually be required to act upon the analysis, such as, teachers, teacher aids, program administrators and any supervisors in the CET program. The needs analysts are those persons responsible for conducting the needs analysis. They may be consultants brought in for the purpose, or members of the faculty designated for the job. The resource group consists of any people who serve as sources of information about the target group. In CET, parents, future employers or professors from the students' content courses may provide valuable information about the target language that students will eventually need to use.

Thirdly, in the process of investigating "needs", different types of questions should be considered, such as, problems, priorities, abilities, attitudes. Problems refer to the problems that the students or the teachers under assessment have to do with and experience in CE learning or teaching. Questions of priority investigate which topics, language uses, skills and so on are considered most important for the students to learn and for the teachers to teach. Ability questions focus on the students themselves who measure their overall English proficiency and to diagnose their specific weaknesses. Attitude questions are created to uncover information about participants' feelings and attitudes toward elements of CE.

Fourthly, the instruments applied in needs analysis contain the following different categories: such as, testing, observations, interview, and questionnaires. Testing is the best way to get the information about the general ability levels of the students. Observations involve watching students' language and classroom behaviors. Interviews include individual and group interviews which allow for gathering personal and group responses and views about CE privately. Questionnaires are more efficient for gathering information on a large scale than the other three.

\subsection{Adaptive CE Teaching Objectives}

In CE, The teaching objective should always adapts to the learners' and the society's needs. As a necessary and important part of curriculum development, the teaching objective is often defined as "the specific statement that describes the particular knowledge, behaviors, and /or skills that the learner will be expected to know or perform at the end of the course or program” [[3], p. 73]. Generally speaking, in curriculum design, there are three kinds of objectives i.e. cognitive, psychomotor and affective objectives. Cognitive objective are concerned with mental or intellectual skills and abilities. Psychomotor objective are concerned with physical skills. Affective objective are concerned with feelings, values and attitudes.

No matter what kinds of objectives they are, the teaching objectives must be adaptive so that they can be tailored to different contexts and adapt to changes over time in the needs of the students or in the physical and human resources of the program. That means the teaching objectives must be established according to the students needs and the social needs, especially, student -oriented. Only by doing this, can educators and teachers clearly know what changes in learning they want students to obtain, what teaching and learning strategies to be chosen to get these changes, inform students of what is expected 
of them in the course, and indicate to them what will be important in evaluating the course and serve as a base line for evaluating the course.

According to College English Curriculum Requirements, the changes of students' learning needs and social needs in China supply us enough evidences to set the teaching objective of CE curriculum like this: CE curriculum must "equip college and university students with an adequate English proficiency, to develop students' ability to use English in a well-rounded way, especially in listening and speaking, so that in their future studies and careers as well as social interactions they will be able to use English effectively in oral and written communication and complete their English-language tasks successfully, and at the same time enhance their ability to study independently and improve their general cultural awareness so as to meet the needs of China's social development and international exchanges” [[7], p. 22].

\subsection{Dynamics College English Testing}

As Bachman points out, “... tests are not developed and used in a value-free psychometric test-tube; they are virtually always intended to serve the needs of individuals and the needs of society at large" [[1], p. 279]. A promising language testing, therefore, should respond as quickly as possible, to students' needs as well as social needs. It should always change all the time with the changes of the world. So we can say that just like a complex dynamic system, CE testing should be dynamic all the time for more appropriate measures of language skills and abilities, and for making decisions about individuals' attained levels of competency with respect to various educational and employment requirements. In $\mathrm{CE}$, recent investigations show that all the students need to have sound competences in practical communication and strong comprehensive applied capacities in their future jobs. So in order to meet such needs, the following work should be done in language testing.

Firstly, special care should be taken to ensure that the test is testing what is intended to test. Therefore, in China, the main task of the language testing designers is to provide a comprehensive assessment of students' communicative language abilities and practical applied capacities.

Secondly, special care should be taken to further improve the language testing's validity and efficiency. For doing this, it is quite important to fully utilize the technological advances in computers and internets.

Thirdly, special care should be taken to develop various large-scale standardized English tests, such as: CET, IELTS, TOEFL and GRE etc. which have great effects on the English teaching and learning in China. Accordingly, great efforts should be made to advocate proper use of those test results by students, teachers, university authorities and other stakeholders, so as to maximize its positive backwash effect on teaching and beneficial impact on society. Further research and discussion should be given to it to improve its validity and efficiency and to make it serve English teaching and learning better.

\subsection{Open CE Teaching Materials}

Teaching materials have one of the features of complex system-openness because CE teaching materials belong to open systems which allow much more advanced teaching materials and information to enter from the outer world and finally become a "far-from-equilibrium" system.

Teaching materials include far more than textbooks, but in China, they are usually equal to textbooks. Because of the conventional heavy dependence on them, textbooks are considered to be one of the major factors affecting English teaching and learning. They are a major source of language input in CET.

However, in China, former English textbooks for nonEnglish major were either inflexible or improper. They were considered to be outdated and not open to all kinds of subjects, writing styles and cultures. Moreover, they often put overemphasis on imparting language knowledge and training language skills without adequate coverage of knowledge in other disciplines.

The openness of teaching materials means that CE textbooks must include articles in different styles, cover a wide range of knowledge, reflect recent developments of science technology and that the textbooks must contain not only British-American cultures but also Chinese and other countries' cultures, which is supposed to effectively improve not only the visual but also the aural and oral comprehension ability of students. The openness of teaching materials also means that textbooks should not be regarded as the only input in CET and that the materials on internets, in newspapers and magazines should be made full use of both in class and outside class.

\subsection{Dynamic and Multiple CE Teaching Models}

In the view of Larsen-Freeman, language is a complex dynamic system, always changing, always adapting and evolving as it is used. Therefore, foreign language learning presents us with an intriguing question. How is a dynamic, constantly changing language to be taught and learned?

CE teaching models should be dynamic because great changes of the society call for the changes in them. Changes in teaching models by no means call for changes in teaching methods and approaches only, but, more important, consist of changes in teaching philosophy and practice, such as, a shift from teacher-centered patterns, in which knowledge of the language and skills are imparted by the teacher in class only, to student-centered patterns, in which the ability to use the language and the ability to learn independently are cultivated, and a change from the fixed and unitary teaching models to a dynamic and multiple ones. The new models can be built on computer and network technology as well as traditional classroom teaching.

In computer-or network-based language teaching or Eteaching, the teaching model should cover the complete process of teaching, learning, feedback and management, including such modules as students' learning and selfassessment, teachers' lectures, and online coaching, as well as the monitoring and management of learning and coaching. It should be able to track down, record and check the progress of learning in addition to teaching and coaching, and attain to a high level of interactivity, multimedia-use and operability. Teachers should adopt good teaching software and make effective use of web multimedia and other teaching resources. 
In traditional classroom teaching, the students' roles should be shifted from passive receivers to active participants. No matter in Basic College English Courses or in Elective College English Courses, practical exercises and activities, such as oral presentations, discussions and debates on hot issues, journalists' interviews and forums, are required in class to realize the interactive functions of the English language. Some principles should be stuck on in English classroom teaching, such as emphasizing students' participation and avoiding unitary class activities, advocating active learning and avoiding passive learning, calling for teacher-student's and student-student's interactions and avoiding teachers' one-way teaching, emphasizing practical application and deemphasizing studying for examinations. All in all, students should be the masters of the classroom teaching and teachers should be the "organizers" of the teaching activities and the “advisors” of students' learning processes. The aims of classroom teaching should pursuit the development of students' comprehensive English competence.

\subsection{Diversified CE Evaluation System}

What kinds of evaluation should be used in the CE curriculum development? The answer is that a diversified evaluation system should be used to evaluate different elements and components of CE curriculum. It is proved that the most effective curriculum evaluation system should comprise formative, summative, and dynamic evaluation.

Formative evaluation refers to procedural and developmental evaluation conducted in the teaching process, i.e., tracking the teaching process, providing feedback and promoting all-round developments of the students, in accordance with the teaching objectives and by means of various evaluative methods. Formative evaluation can take such forms as keeping a record of students' in and outside of classroom activities and online self-learning data, keeping files on students' study results, and conducting interviews and holding meetings. This allows students' learning processes to be subjected to observation, evaluation and supervision, thus contributing to the enhancement of their learning efficiency.

Summative evaluation is conducted at the end of a teaching phase. It mainly consists of final tests and proficiency tests, designed to evaluate student's all-round ability to use English. These tests aim to assess not only students' competence in reading, writing and translation, but also their competence in listening and speaking. To make a summative evaluation of teaching, colleges and universities may administer tests of their own, or let students take the national or international tests after meeting different standards set by the College English Curriculum Requirements [7]. Whatever form the tests may take, the focus should be on the evaluation of students' abilities to use English in communication, particularly their ability to listen and speak in English.

Dynamic Evaluation (DA): Dynamic evaluation is grounded in Vygotsky's important insight that in the ZPD (the Zone of Proximal Development) instruction leads development [18]. It is a most advanced evaluation system with the aim to assess an individual's learning potential because it is not enough to know what an individual can do alone without assistance, as reflected in traditional approaches to testing and it is necessary to discover what the person can do through scaffolding (i.e., instruction, peer supports or learning aids etc..), because it portrays what the person will eventually be able to do when that help has been internalized.

Unlike other evaluations, which set their objective as evaluating the level of students or providing feedback for their present study, DA lays special emphasis on students' future cognitive abilities, potential developments, In fact, DA is an interactive approach to psychological assessment that embeds intervention within the assessment procedure. By interpreting the potential of learners (pre-task), assistance is made via the interaction between mediators and learners aided by language (dialogue) or other meditational tools (mediation). Since the mediation is made in the Zone of Proximal Development of learners, remarkable progress is likely to occur.

\section{Conclusion}

Complex System Theory, as a frontier theory in Applied Linguistic, has changed the viewpoints about language nature, language development, and language classroom teaching, which brought pedagogical implication to CET development in China. Teachers should see CET procedure as a dynamic, open, adaptive, multi-level interactive and non-linear system and try to explore the effective approaches to CET development under the theory. Only by doing this, can CET really reach such aims as to help students develop a relatively high level of ability in reading and general skills of listening, speaking, writing and translation so that they can communicate effectively in English and help students lay a solid language foundation, grasp effective language learning methodologies, and nourish cultural accomplishment so that they can meet the needs of social development and economic growth. And meanwhile, in this way, Complex System Theory can draw much more attention, be developed greatly and go across much more disciplines in China.

\section{Acknowledgement}

This paper was sponsored by the Teaching Program of University of Jinan (JZ1320).

\section{References}

[1] Bachman, L. F, Fundamental Considerations in Language Testing, Oxford University Press, Oxford, 1990, 279.

[2] Brown, J. D, The Elements of Language Curriculum: A Systematic Approach to Program Development, Heinle and Heinle, Boston, 1995, 29-35.

[3] Brown, D. H, Teaching by Principle: A Interactive Approach to Language Pedagogy, Foreign Language Teaching and Research Press, Beijing, 2001, 73.

[4] DeBot, K. "Introduction: Second language development as a dynamic process," The Modern Language Journal, Volume 92, 166-178, 2008.

[5] De Bot, K., W. Lowie \& M. Verspoor, Second Language Acquisition: An Advanced Resource Book, Routledge, London, 2005, 3-27. 
[6] De Bot, K.,W. Lowie \& M. Verspoor, "Dynamic Systems Theory approach to second language acquisition,” Bilingualism, Volume 10 (1). 7-21. 2007.

[7] Education Department of China, College English Curriculum Requirements (for Formal Implementation), Shanghai Foreign Languages Education Press. Shanghai, 2007, 22-257.

[8] Larsen-Freeman,D, "Chaos/Complexity science and second language acquisition,” Applied Linguistics, Volume 18. 141-165. 1997.

[9] Larsen-Freeman, D, "Language acquisition and language use from a chaos/complexity theory perspective," In C. Kramsch (ed.). Language Acquisition and Language Socialization, Continuum, London, 2002, 33-46.

[10] Larsen-Freeman, D, "The emergence of complexity, fluency, and accuracy in the oral and written production of five Chinese learners of English," Applied Linguistics, Volume 27. 590-619. 2006.

[11] Larsen-Freeman, D, "On the complementarity of Chaos/Complexity Theory and Dynamic Systems Theory in understanding the second language acquisition process," Bilingualism, Volume 10. 35-37. 2007.
[12] Larsen-Freeman, D.\&L. Cameron, Complex Systems and Applied Linguistics, Oxford University Press, Oxford ,2008, 25-98.

[13] Larsen-Freeman, D, "Adjusting expectations: The study of complexity, accuracy, and fluency in second language acquisition,” Applied Linguistics, Volume 30. 579-589. 2009.

[14] Larsen-Freeman, D\&L. Cameron, "Research methodology on language development from a Complex Systems perspective,” The Modern Language Journal, Volume 92. 200-213. 2008.

[15] Meara, P, "Towards a new approach to modeling vocabulary acquisition," In N. Schmitt and M. McCarthy (eds.). Vocabulary: Description, Acquisition and Pedagogy, Cambridge University Press, Cambridege, 1997.115.

[16] Van Geert, P. "Dynamic Systems approaches and modeling of developmental processes,” In J. Valsiner \& J. Conlolly (eds.). Handbook of Developmental Psychology, Sage London, 2003, 640-672.

[17] Van Geert,P. "The dynamic systems approach in the study of L1 and L2 acquisition: An introduction," The Modern Language Journal, Volume 92.179-199.2008.

[18] Vygotsky, L. Mind in society: The development of higher psychological processes, Harvard University Press, Cambridge, MA, 1978, 56-58. 